\title{
insideOutside: an accessible algorithm for classifying interior and exterior points, with applications in embryology
}

\author{
Stanley E. Strawbridge ${ }^{1,2, *}$, Agata Kurowski ${ }^{3}$, Elena Corujo-Simon ${ }^{1,2,4}$, Alexander G. \\ Fletcher ${ }^{5,6, *}$, Jennifer Nichols ${ }^{1,2,4,7, *}$ \\ ${ }^{1}$ Wellcome-MRC Cambridge Stem Cell Institute, University of Cambridge, Cambridge, UK. \\ ${ }^{2}$ Department of Physiology, Neuroscience and Development, University of Cambridge, Cambridge, UK. \\ ${ }^{3}$ Department of Pharmacological Sciences, Icahn School of Medicine at Mount Sinai, New York, NY, USA. \\ ${ }^{4}$ MRC Human Genetics Unit, University of Edinburgh, Edinburgh, UK. \\ ${ }^{5}$ School of Mathematics and Statistics, University of Sheffield, Sheffield, UK. \\ ${ }^{6}$ The Bateson Centre, University of Sheffield, Sheffield, UK. \\ ${ }^{7}$ Centre for Trophoblast Research, University of Cambridge, Cambridge, UK. \\ * E-mail: ss2199@cam.ac.uk, a.g.fletcher@sheffield.ac.uk, jenny.nichols@ed.ac.uk
}

\section{Key Words}

Machine Learning; Quantitative Biology; Pre-implantation; Embryo; Inner Cell Mass;

Trophectoderm.

\begin{abstract}
A crucial aspect of embryology is relating the position of individual cells to the broader geometry of the embryo. A classic example can be seen in the first cell-fate decision of the mouse embryo, where interior cells become inner cell mass and exterior cells become trophectoderm. Advances in image acquisition and processing technology used by quantitative immunofluorescence have resulted in the production of embryo images with increasingly rich spatial information that demand accessible analytical methods. Here, we describe a simple mathematical framework and an unsupervised machine learning approach for classifying interior and exterior points of a three-dimensional point-cloud. We benchmark our method to demonstrate that it yields higher classification rates for pre-implantation mouse embryos and greater accuracy when challenged with local surface concavities. This method should prove useful to experimentalists within and beyond embryology, with broader applications in the biological and life sciences.
\end{abstract}




\section{Introduction}

The mouse embryo undergoes three major morphogenetic events between fertilization and implantation, termed: compaction, cavitation, and hatching (Figure1A,B) (Tarkowski and Wróblewska, 1967, Smith and McLaren, 1977; Yoshinaga et al., 1976). Compaction coincides with the first binary cell-fate decision, which is ultimately driven by position within the embryo (Figure1C) (Tarkowski and Wróblewska, 1967). Exterior cells polarize to become the extraembryonic trophectoderm (TE), placental precursor (Lawson et al., 1999), while the interior cells become the inner cell mass (ICM) (Ziomek and Johnson, 1980, Johnson and Ziomek, 1981). The ICM cells undergo a second binary cell-fate decision to become either the embryonic epiblast, source of the foetus (Gardner and Rossant, 1979) and embryonic stem cells (Evans and Kaufman, 1981, Martin, 1981), or the primitive endoderm (PrE), founder of the yolk sac (Gardner and Johnson, 1972). This second cell-fate decision coincides with cavitation, where a fluid-filled cavity, called the blastocoel, forms between the TE and one side of the ICM (Smith and McLaren, 1977). Finally, prior to implantation, the embryo must hatch. This is the process where the embryo will break out of and dissociate from the zona pellucida (Malter and Cohen, 1989, Suzuki et al. 1995; Yoshinaga et al., 1976).

Molecular profiling of these tissues has been performed at the levels of RNA-sequencing (Boroviak et al., 2018, Guo et al., 2010, 2017, Kurimoto et al., 2006) and immunohistochemistry (Chazaud et al., 2006; Niwa et al., 2005; Palmieri et al., 1994; Plusa et al., 2008) to reveal key lineagemarkers. These lineage-markers have been used to study the dynamic emergence and plasticity of distinct cell-identities throughout the stages of pre-implantation development by employing fluorescent reporter knock-ins (Arnold et al., 2011; Grabarek et al., 2012; Hamilton et al., 2003, Kalkan et al., 2017, McDole and Zheng, 2012, Plusa et al., 2008). However, there remain instances where reliable lineage-markers do not exist. For example, in unspecified cells of the compacting morula, where a single cell can co-express lineage-markers for TE (CDX2), PrE (GATA6), and epiblast (SOX2) (Plusa et al., 2008). There are also instances in genetic knockouts (KOs) where lineage-markers no longer faithfully mark their tissue. In the example of the pluripotency factor Oct4, expression levels of early ICM lineage-markers KLF4 and TFCP2L1 are significantly lower in KO mice than that of wild-type mice at the mid-blastocyst stage Stirparo et al. 2021). Additionally, Oct4-KO embryos exhibit lower levels of expression in early PrE marker GATA6 and fail to initiate expression of late PrE marker Sox17 (Le Bin et al., 2014). A more severe case can be seen in the KO of the early PrE marker Gata6, where embryos not only failed to activate the expression of other PrE markers, PDGFRA, SOX17 and GATA4, but also expressed the ICM marker NANOG in the TE (Bessonnard et al., 2014; Schrode et al., 2014). Finally, moving beyond the mouse, definitive sets of lineage-markers have yet to be firmly established for other mammalian systems, such as human and non-human primates (Boroviak et al., 2018; Guo et al., 2021; Stirparo et al., 2018). In such instances as these, we must find alternative methods to classify the tissues we wish to investigate.

Positional information is a classical method for classifying cell populations in the preimplantation mouse embryo (Fleming, 1987; Nichols and Gardner, 1984). Advances in both 
A
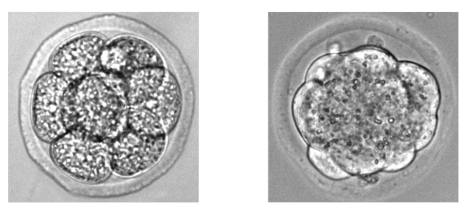

$50 \mu \mathrm{m}$

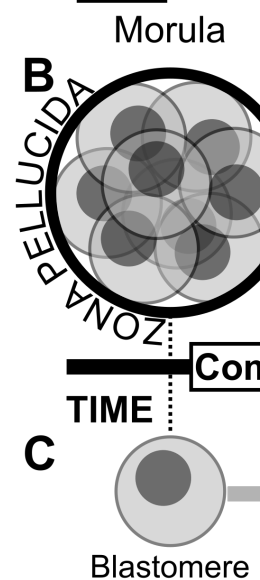

Compacted Morula
Blastomere

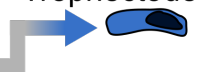

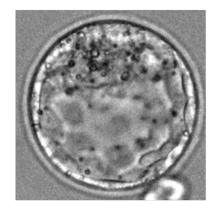

Mid Blastocyst

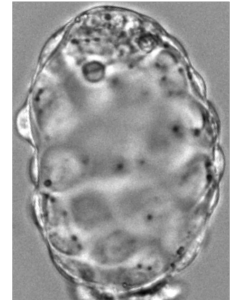

Late Blastocyst
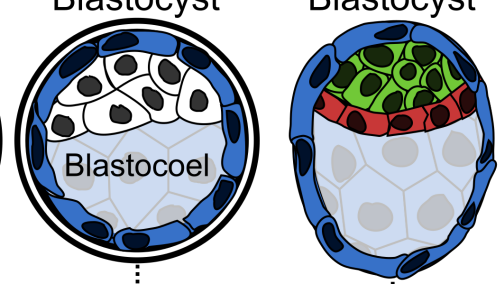

Hatching

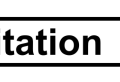

Trophectoderm
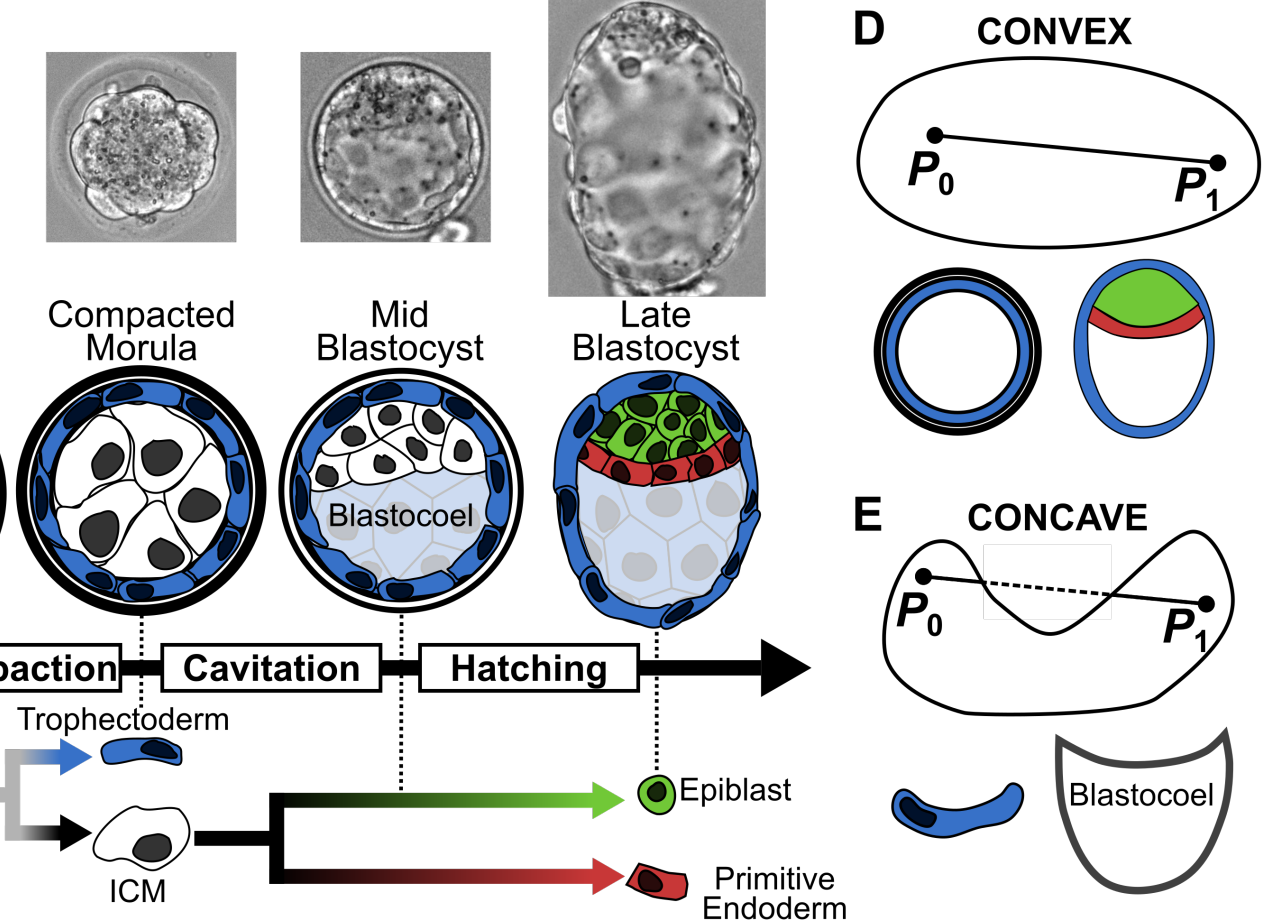

E

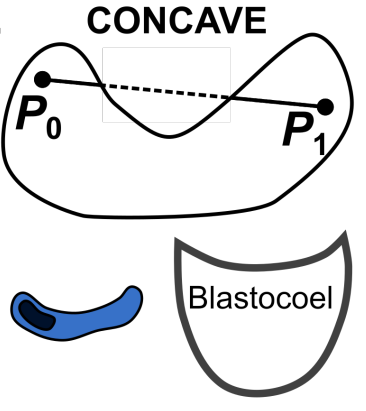

Figure 1: A. Bright-field images from pre-implantation mouse development, from morula to late blastocyst. B. Three major morphogenetic events occur during pre-implantation mouse development: compaction, cavitation, and hatching. C. Cells in the pre-implantation embryo make two sets of binary cell-fate decisions: first, blastomeres become inner cell mass (ICM) (interior) or trophectoderm (exterior); second, ICM become epiblast or primitive endoderm. These decisions coincide with compaction and cavitation, respectively, and are completed by hatching. D. A shape is convex if for any pair of points, $P_{0}$ and $P_{1}$, the resulting line segment is entirely contained within the shape; biological examples of convex shapes include the compacted morula and blastocyst. E. A shape is concave if there exists at least one pair of points whose resulting line segment passes to the exterior of the shape; biological examples of concave shapes include trophectoderm cells and the blastocoel cavity.

image acquisition and image processing technologies have improved the accuracy of this positional information. A common analysis method in mouse embryology involves quantitative immunofluorescence (qIF) of cellular nuclei. This is the process in which three-dimensional (3D) confocal fluorescence microscopy images of nuclei are segmented and quantified using software such as FIJI (Schindelin et al., 2012), MINS (Lou et al., 2014), or Nysses (Blin et al., 2019). Output parameters from qIF may include total nuclear fluorescence, nuclear volume, and the geometric center (centroid) of the nucleus. These centroids can then be used to classify nuclei by their relative position within the resulting point-cloud. Classification of interior and exterior nuclei are of particular interest when investigating the relationship between the cells of the ICM (interior) and the TE (exterior).

To date, three methods have been used to classify interior and exterior nuclei of the mouse embryo from qIF. We refer to these as the Ellipsoidal, Convex Hull, and insideOutside methods. The Ellipsoidal method, employed by the nuclear segmentation software MINS (Lou et al., 2014), fits an ellipsoid to the point-cloud generated by segmented nuclear centroids. A nucleus is then 
classified as exterior if the distance from the ellipsoid's centre to the nuclear centroid exceeds 0.95 times the distance from the ellipsoid's centre to the point on the ellipsoid that is closest to the nucleus's centroid; otherwise, the cell is classified as interior. The MINS software package has been widely used for qIF and has been cited in nearly 100 manuscripts. The Convex Hull method, employed by the spatial analysis software IVEN (Forsyth et al., 2021), constructs a convex hull, the smallest convex set that contains all centroids, from all nuclear centroids of the embryo and then classifies a nucleus as exterior if it belongs to the boundary of the convex hull. IVEN does allow for manual correction of the classification, however, this requires user input which may introduce bias. The insideOutside method, employed by Stirparo et al. (2021) and the focus of the present work, is an accessible position-based approach to the classification of interior and exterior nuclei. Here we use unsupervised classification over two parameters that relate a nuclear centroid to the boundary of the convex hull generated from all nuclear centroids of the embryo.

These methods share the common assumption that the embryo is convex. A shape is said to be convex if the line segment between any pair of points within the shape is entirely contained within the shape (Figure 1D); otherwise the shape is said to be concave (Figure1E). The Ellipsoidal method treats the embryo as an ellipsoid, which is a convex shape. Similarly, the Convex Hull method explicitly defines the exterior points as being a member of a convex shape. Therefore, these methods underperform if the surface of the embryo exhibits small local concavities or if the embryo incurs indentations through fixation and mounting. The insideOutside method softens the assumption of convexity by classifying points using a two-dimensional parameter space instead of requiring strict membership of a convex shape.

We finish by noting that it has become increasingly important for experimentalists to perform rigorous quantification of the data they generate. Thus it is necessary to develop easy-to-deploy software that does not require high levels of programming expertise. Furthermore, benchmarking of the three mentioned classification methods has yet to be performed. Therefore, we present the accessible insideOutside algorithm for the classification of interior and exterior points of a relatively convex shape. We detail the mathematical framework underpinning the twodimensional parameter space used for unsupervised classification, along with accuracy testing. We then proceed to benchmark the three classification methods (Ellipsoidal, Convex Hull, and insideOutside) using pre-implantation mouse blastocysts, showing that the Convex Hull and insideOutside methods outperform the Ellipsoidal method. We conclude by demonstrating that the insideOutside method outperforms the Convex Hull method when challenged with local surface concavities, similar to what would be found in empirical data sets. 
bioRxiv preprint doi: https://doi.org/10.1101/2021.11.15.468285; this version posted November 17, 2021. The copyright holder for this preprint (which was not certified by peer review) is the author/funder, who has granted bioRxiv a license to display the preprint in perpetuity. It is made available under aCC-BY-NC-ND 4.0 International license.

\section{Results}

The minimum distance and variance in distances from a point to the surface of a convex shape are inversely related

Here we will establish minimum distance to the surface and the variance in all distances to the surface of a convex shape as the parameters used for the insideOutside algorithm. An intuitive understanding for the selection of these parameters follows by considering what happens to these two parameters for two different points within a sphere: a point at the centre and a point at the surface.

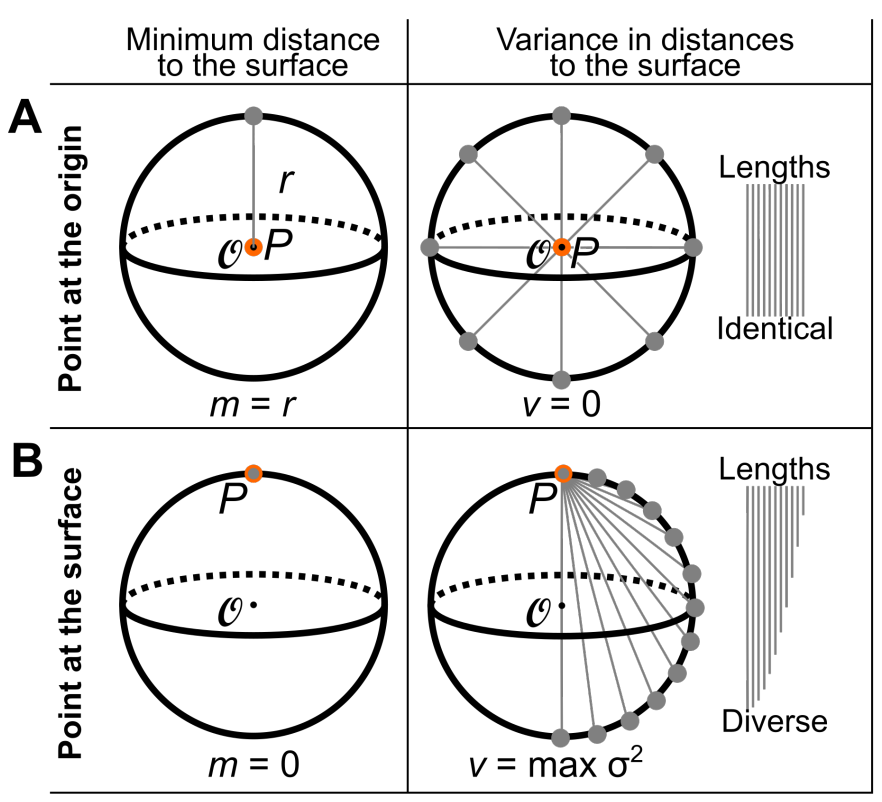

\section{For an arbitrary point within a sphere Minimum distance to the surface}

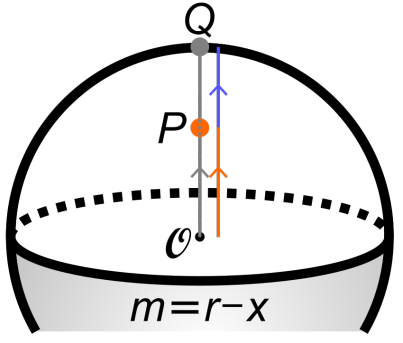

Variance in distances to the surface
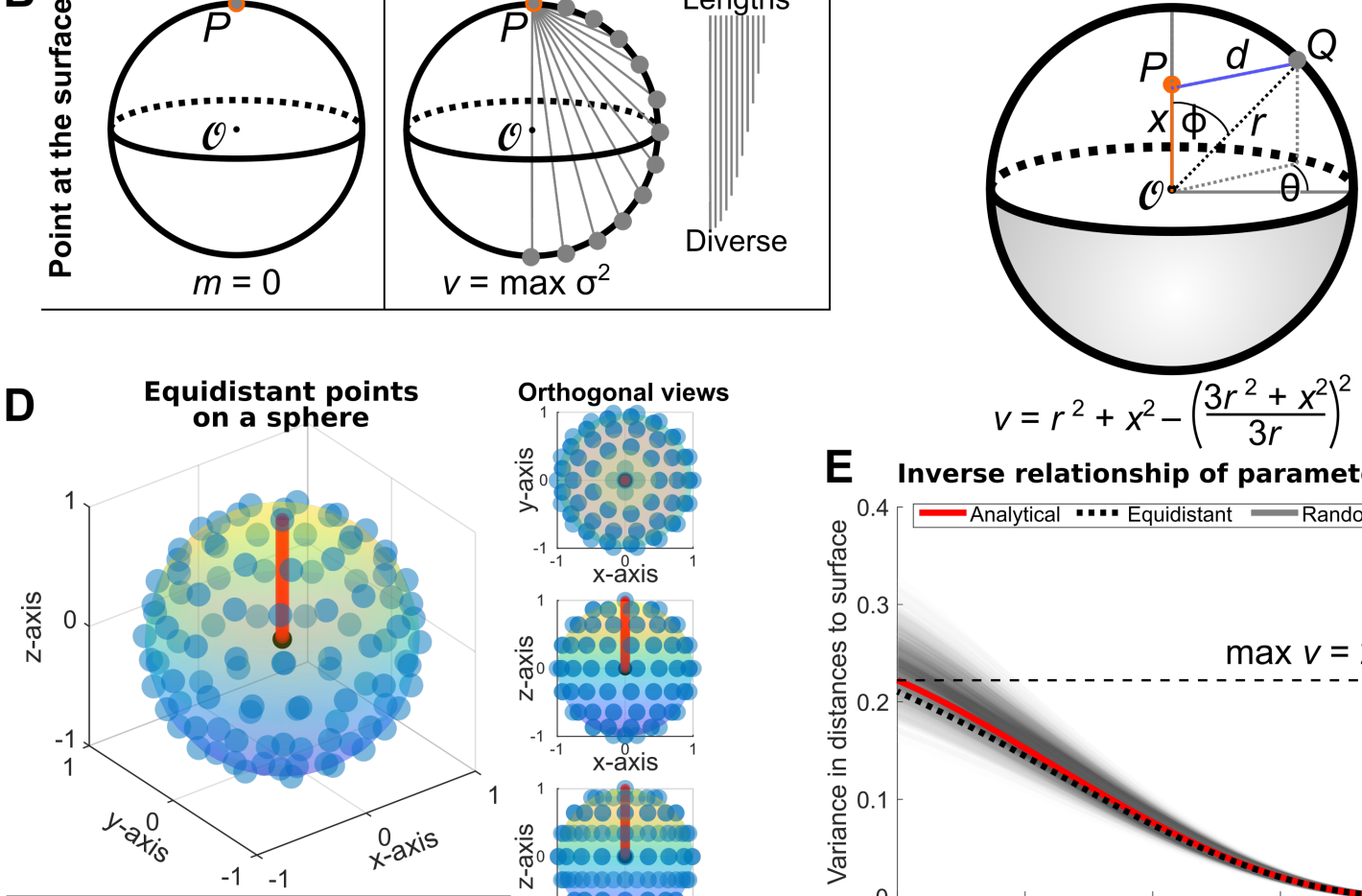

Surface points๑ Test points• Origin

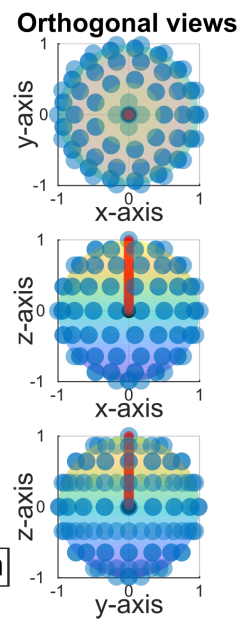

E Inverse relationship of parameters

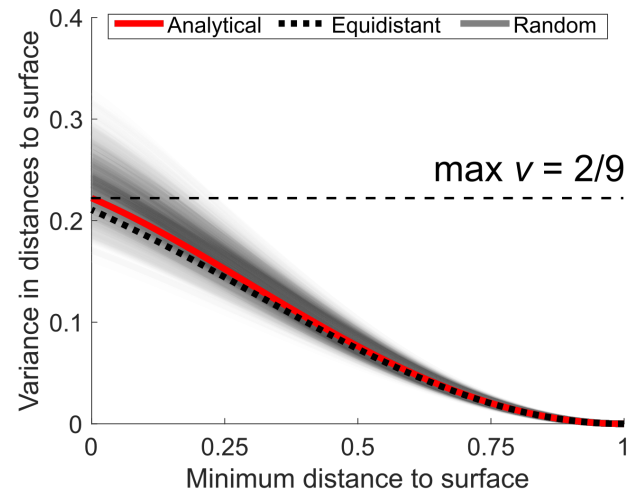

Figure 2: A. For a point $P$ (orange dot) coincident with the centre of a sphere $(\mathcal{O}$, black dot) of radius $r$, the minimum distance from $P$ to the sphere is $r$ and the variance in distances is zero. B. For a point $P$ located on the sphere, the minimum distance from a point $P$ to the sphere is zero, and the variance in distances is greatest. C. Analytic expressions for the minimum distance, $m$, and variance in distances, $v$, from a point $P$ located on/inside a sphere of radius $r$ centred at the origin to the sphere. $\mathbf{D}$. This 
relationship is tested for spheres that are discretized using equidistant points. 100 equidistant points (blue dots) are plotted on the unit sphere (rainbow surface). $m$ and $v$ are calculated for 50 test points (red dots) along the vector from the origin (black dot) to the surface point $P=[0,0,1]$. Shown are the three-quarters view (left) and the three orthogonal views (right). E. The inverse relationship between $m$ and $v$ are shown for the continuous case of the unit sphere (see (5) (red line) and discrete cases of 100 equidistant points on the unit sphere (Figure 2D, black dotted line) and 100 uniform random points on the unit sphere (Figure S1, translucent black lines, 1000 realizations).

First, let us consider what happens to these parameters for the point at the exact centre of the sphere (Figure 2A). The minimum distance to the sphere is exactly the radius of the sphere. In fact, the distance from the centre to all other points of the sphere are identically the radius, meaning that the variance in distance to the surface of the sphere is exactly zero. Thus, minimum distance to the surface is maximized and the variance in distances is minimized for the point at the centre of a sphere.

On the other hand, consider an arbitrary point on the surface of that same sphere (Figure $2 \mathrm{~B}$ ). For that surface point, the minimum distance to the surface of the sphere is exactly zero. If we then draw line segments from that point to all other points on the surface of the sphere, we see that we are drawing line segments of every length between zero and the diameter of the sphere. Meaning that the original point on the surface of the sphere achieves the most diversity of line segment lengths possible for the sphere. In other words, a point on the surface of the sphere has the maximum variance in distances to the surface. Thus, minimum distance to the surface is minimized and the variance in distances to the surface has been maximized for any point on the surface of a sphere. We therefore arrive at an inverse relationship between the minimum distance and the variance in distances to the surface of a sphere as we move from the centre of the sphere to the surface of the sphere.

We now formalize this relationship. First, we derive the expression for the minimum distance from any point on/inside the sphere of radius $r$, centred at the origin, to the sphere. Intuitively, a point $P$ on/inside the sphere, its closest point on the sphere, and the origin all lie on a straight line (Figure $2 \mathrm{C}$, top). Hence, if $P$ is located a distance $x \in[0, r]$ from the origin, then since the distance from any point on the sphere to the origin is $r$, the minimum distance from $P$ to the sphere is given by $m=r-x$.

Next, we derive the expression for the variance in distances from a given point on/inside the sphere to the sphere. Let $P$ be located at distance $x \in[0, r]$ from the origin, and without loss of generality let $P$ lie on the $y$-axis. Consider a point $Q$ on the sphere, whose angle to the $y$-axis is given by $\phi$ (Figure $2 \mathrm{C}$, bottom). Since the distance from $Q$ to the origin is $r$, by the law of cosines the distance from $P$ to $Q$ is given by $d(\phi)=\sqrt{r^{2}+x^{2}-2 r x \cos \phi}$. The variance in distances from $P$ to the sphere is thus given by,

$$
v=\mathbb{E}\left[d^{2}\right]-\mathbb{E}[d]^{2}
$$


Computing surface integrals, we find that the mean distance $\mathbb{E}[d]$ is given by,

$$
\mathbb{E}[d]=\frac{1}{4 \pi r^{2}} \int_{\phi=0}^{\pi} \int_{\theta=0}^{2 \pi} d(\phi) r^{2} \sin \phi \mathrm{d} \theta \mathrm{d} \phi=\frac{3 r^{2}+x^{2}}{3 r}
$$

while the second moment $\mathbb{E}\left[d^{2}\right]$ is given by,

$$
\mathbb{E}\left[d^{2}\right]=\frac{1}{4 \pi r^{2}} \int_{\phi=0}^{\pi} \int_{\theta=0}^{2 \pi}(d(\phi))^{2} r^{2} \sin \phi \mathrm{d} \theta \mathrm{d} \phi=r^{2}+x^{2},
$$

hence the variance in distances is given by,

$$
v=r^{2}+x^{2}-\left(\frac{3 r^{2}+x^{2}}{3 r}\right)^{2} .
$$

We next show that the minimum distance and variance in distances to the sphere are inversely related. Substituting $x=r-m$ into our expression for $v$, after some algebra we obtain,

$$
v=-\frac{1}{9 r^{2}}(m-r)^{2}\left(m^{2}-2 m r-2 r^{2}\right),
$$

from which we obtain,

$$
\frac{d v}{d m}=-\frac{2}{9 r^{2}}(m-r)\left(2 m^{2}-4 m r-r^{2}\right)
$$

Since $m-r \leq 0$ and $2 m^{2}-4 m r-r^{2}<0$ for $m \in[0, r]$, we have $d v / d m \leq 0$ for $m \in[0, r]$, hence $v$ is a decreasing function of $m$ for $m \in[0, r]$. Thus, for points on/inside the sphere, the variance in distances is inversely related to the minimum distance to the sphere. Finally this relationship can be seen by plotting (5) for the unit sphere $(r=1)$ (Figure 2 $\mathrm{E}$ ).

We conclude this section by showing that this inverse relationship holds for a convex surface generated by a set of discrete points. For this we simulate 100 points on the surface of a sphere that are spaced either equidistant (Figure 2D) or uniformly at random (1000 realizations) (Figure S1) (Deserno, 2004). For each discrete surface, the minimum distance and variance in distances to the surface points are calculated for 50 test points equally spaced between the centre of the sphere and a surface point. Both sets of simulations closely match the analytical solution (Figure 2 $\mathrm{E}$ ). While this has been demonstrated for the case of the sphere and discretized derivatives of the sphere, it provides a theoretical foundation for the use of $m$ and $v$ in the classification of convex point-clouds. Importantly, the demonstration using discretized surfaces indicates that this relation is directly applicable to empirical data which consist of discrete points, e.g. the centroids of cellular nuclei within an embryo. 
bioRxiv preprint doi: https://doi.org/10.1101/2021.11.15.468285; this version posted November 17, 2021. The copyright holder for this preprint (which was not certified by peer review) is the author/funder, who has granted bioRxiv a license to display the preprint in perpetuity. It is made available under aCC-BY-NC-ND 4.0 International license.

\section{insideOutside: a two-dimensional decision space for classifying interior and exterior positions}

Motivated by the theoretical result of the previous section, we proceed to describe an algorithm for the classification of interior and exterior points of a 3D point-cloud. The insideOutside algorithm (Algorithm 1) takes in a set of 3D Cartesian coordinates, $S \in \mathbb{R}^{m \times 3}$ (Figure 3Ai), and returns indexing vector $I \in \mathbb{B}^{m}$ with 0 indexing the inside points and 1 indexing the outside points. For pre-implantation embryos, the input data can be generated through manual nuclear segmentation in Fiji (Schindelin et al., 2012) or MATLAB's volumeSegmenter App (Copyright 2020 The MathWorks, Inc) or through automated 3D nuclear segmentation pipelines like MINS (Lou et al., 2014) or Nessys (Blin et al., 2019). The algorithm begins by computing the Delaunay triangulation, $\mathcal{D}$, over $S$ (Figure 3 Aii). From $\mathcal{D}$, we generate a convex hull (Barber et al. 1996) (Figure 3Aiii). Now, using $\mathcal{H}$ we can calculate the distance function, $d(P, \mathcal{H}), \forall P \in S$ (Figure 3Aiv). We calculate the minimum and variance in $d(P, \mathcal{H}), \forall P \in S$, and then scale each parameter such that the maximum is 1 and the minimum is 0 (Figure $3 \mathrm{Av}$ ). Finally, hierarchical clustering by ward linkage is performed on the parameters to classify the points into two groups (Figure 3Avi,vii).

Algorithm 1: insideOutside takes in an $n \times 3$ matrix of Cartesian points and returns a bit vector that classifies each point as either inside, 0 , or outside, 1.

Data: Set of points $S \in \mathbb{R}^{m \times 3}$

Result: Classification vector $I \in \mathbb{B}^{m}$

Delaunay triangulation $\mathcal{D}$ over $S$

Generate convex hull $\mathcal{H}$ from $\mathcal{D}$

for each point $P \in S$ do

for each face $f \in \mathcal{H}$ do

| Calculate min $d(P, f)$

end

Calculate $m=\min d(P, \mathcal{H})$

Calculate $v=\operatorname{Var}(d(P, \mathcal{H}))$

end

Perform unsupervised classification for two groups

The accuracy of the insideOutside method classification was performed on test shapes designed to resemble the late mouse blastocyst, whose cell number ranges between 100-150 (Plusa) et al. 2008), where approximately $60-70 \%$ of the cells belong to the TE (outside cells) (Fleming, 1987; Saiz et al., 2016, 2020; Morgani et al., 2018). Therefore we constructed shapes with 100 uniform random points on the unit sphere (outside) and 50 uniform random points within balls of radii between 0.01 and 1 (inside), both centered at the origin (Figure $3 \mathrm{~B}$ and Figure S2). 1000 shapes were simulated and classified for each of 100 inner ball radii.

Initial tests revealed near-perfect classification rates for True Outside points at all inner ball radii (Figure $3 \mathrm{C}$ ). There was, however, a significant drop in the True Inside classification rate at an inner ball radius of 0.82 where the True Inside classification rate dropped below 0.99 with minimum rate of $0.48 \pm 0.17$ (mean \pm standard deviation) at an inner ball radius of 1 . To 
A

A i

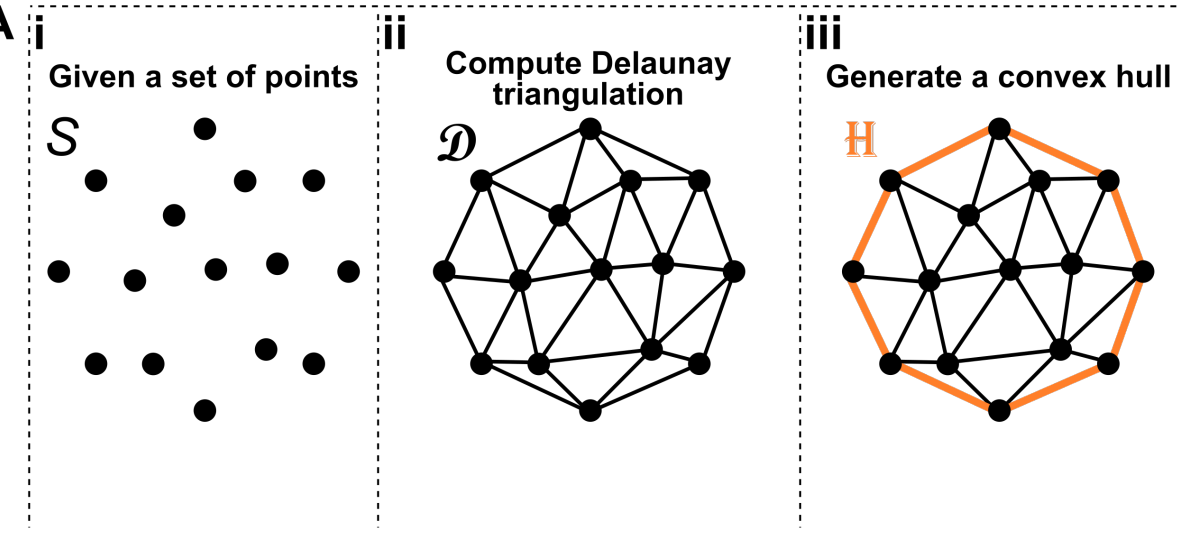

iv

Calculate distance

to each face of hull

H

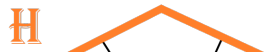

Valculate parameter space
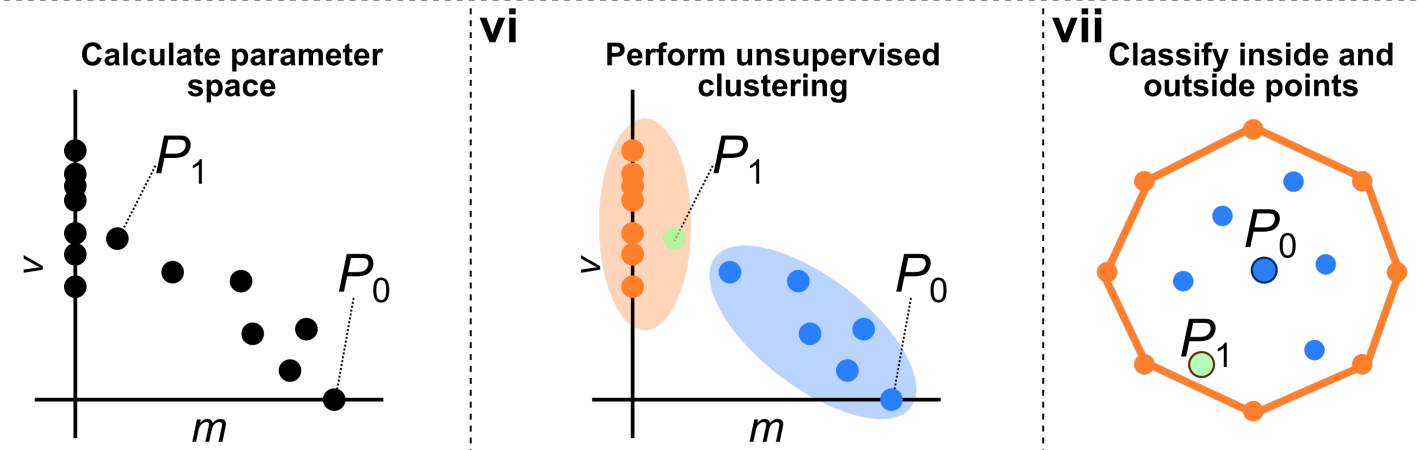

B

i Ground truth

ii Triangulated

iv Classification hull
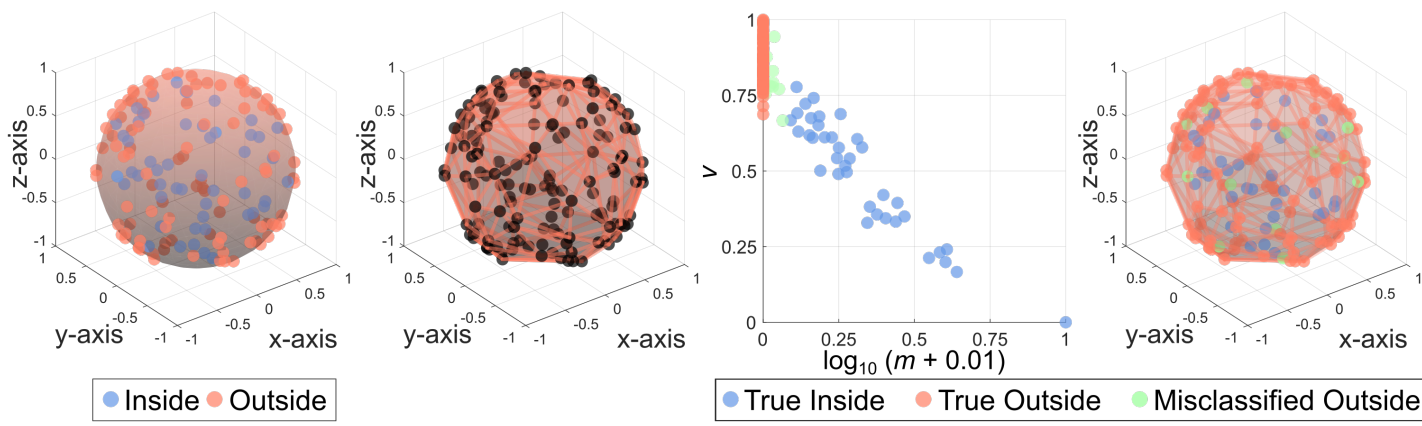

- True Inside True Outside Misclassified Outside

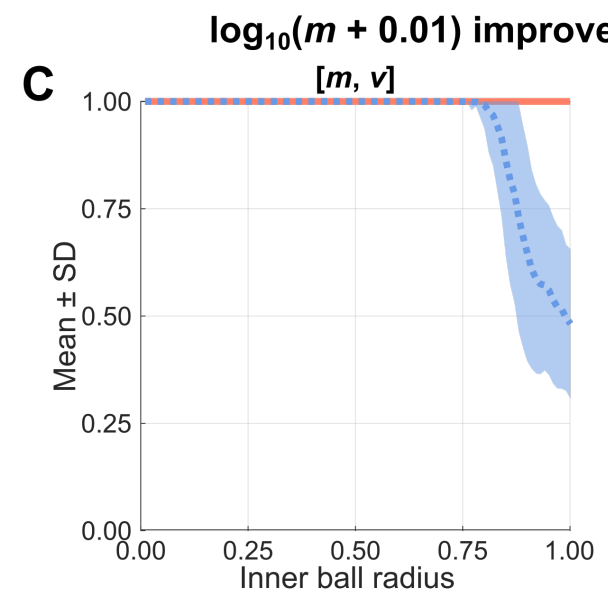

es accuracy of inside classification

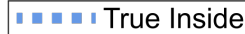

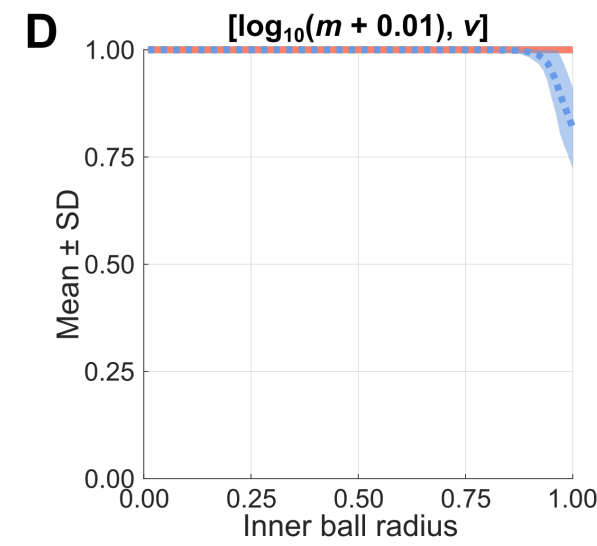

True Outside

Figure 3: A. Outline of the insideOutside algorithm. B. Accuracy testing was performed on a shape 
constructed of 100 outside points, uniform random points on the unit sphere, and 50 inside points, uniform random points in a ball centered at $\mathcal{O}$ of radii ranging from 0.01 to 1 (see Figure $\mathrm{S} 2$ ). Bi-iv. The steps of the algorithm performed on an example shape with inner ball radius of 1 . Bi. Ground truth of inside points, blue dots enclosed by blue surface, and outside points, orange points on orange surface. Bii. The triangulated hull generated from making a convex hull over the Delaunay triangulation. Biii. The classification of points using hierarchical clustering over the calculated parameter space. Shown are True Inside points (blue), True Outside points (orange), and Misclassified Outside points (green). Biv. The classification mapped onto the original shape. C-D. Accuracy testing was performed by classifying the points of 1000 shapes for each of 100 different inner ball radii. The mean True Inside rate (blue dotted line) is shown with standard deviation (blue filled region) and the mean True Outside rate (orange solid line) is shown with standard deviation (orange filled region). C. Accuracy test for the parameters $m$ and $v$. D. Accuracy test for the parameters $\log _{10}(m+0.01)$ and $v$.

improve the True Inside classification rate, we modified the parameter space by taking the log of $m$ (Figure 3D). Simulations bear out marked improvements in True Inside classification rates with no detriment to True Outside classification rates. The resulting True Inside classification rates do not drop below 0.99 until an inner ball radius of 0.94 and achieve a minimum of only 0.82 \pm 0.09 at an inner ball radius of 1 . Thus we have established our algorithm using the parameters of $\left[\log _{10}(m+0.01), v\right]$ and we now proceed to challenge it with empirical data.

\section{insideOutside and Convex Hull methods outperform the Ellipsoidal method when classifying cells of the mouse blastocyst}

In this section we set out to show that the insideOutside method can successfully classify the nuclei of real world embryos. In doing so, we also benchmark our method against two other methods (Lou et al., 2014; Forsyth et al., 2021) using previously quantified mouse midblastocysts (Stirparo et al. 2021) (Figure 4A). SOX2 staining, which can be used to mark all nuclei of the early ICM (Wicklow et al., 2014), were used as the ground truth for benchmarking, where SOX2 positive nuclei indicate inside nuclei and SOX2 negative nuclei indicate outside nuclei. Sox2 positive/negative status was determined through statistical inference (Gaussian mixture modelling) with 758 cells from 14 embryos (Figure 4B,C). We then used the SOX2 ground truth (Figure 4D) to calculate the True Inside and True Outside rates for the three classification methods: Ellipsoidal (Lou et al., 2014), Convex Hull (Forsyth et al., 2021), and insideOutside (Stirparo et al., 2021) (Figure $4 \mathrm{E}$ ).

For inside nuclei classification (Figure $4 \mathrm{~F}$,left), we find that both the Convex Hull (rate = $0.92 \pm 0.06$, mean \pm standard deviation) and insideOutside (rate $=0.91 \pm 0.06$ ) methods outperform the Ellipsoidal method (rate $=0.62 \pm 0.12)\left(\mathrm{p}\right.$-value $=0.8 \times 10^{-4}$ and $1.3 \times 10^{-4}$ respectively, Kruskal-Wallis). While the Convex Hull and insideOutside methods show no difference in ability to classify inside nuclei ( $\mathrm{p}$-value $=0.99$, Kruskal-Wallis). For outside nuclei classification (Figure $4 \mathrm{~F}$,right), we see no difference between the three methods, with classification rates of $0.94 \pm 0.06$ for the Ellipsoidal method, $0.89 \pm 0.09$ for the Convex Hull method, and $0.90 \pm 0.09$ for the insideOutside method (all pair-wise p-values $\geq 0.63$, Kruskal-Wallis). 
A

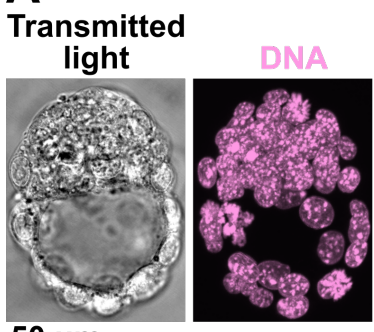

$50 \mu \mathrm{m}$
BGaussian mixture modelling (GMM) of normalized fluorescence
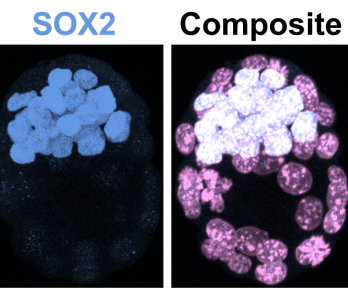
1.00

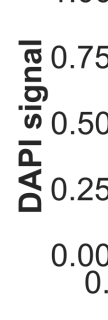

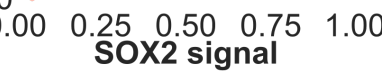

C

Tissue classification by SOX2 signal

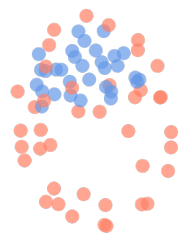

D Ground truth

E

SOX2 positive SOX2 negative SOX2 GMM Ellipsoidal

Benchmarking

Convex Hull insideOutside

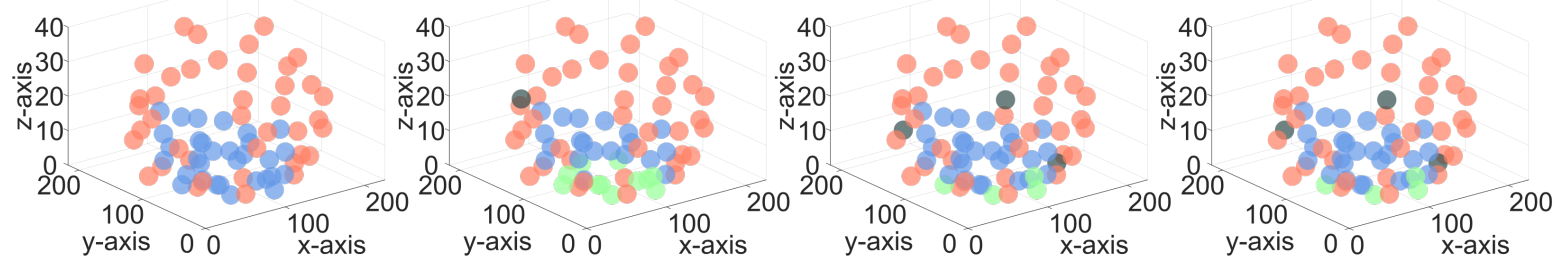

True Inside True Outside Misclassified Outside Misclassified Inside
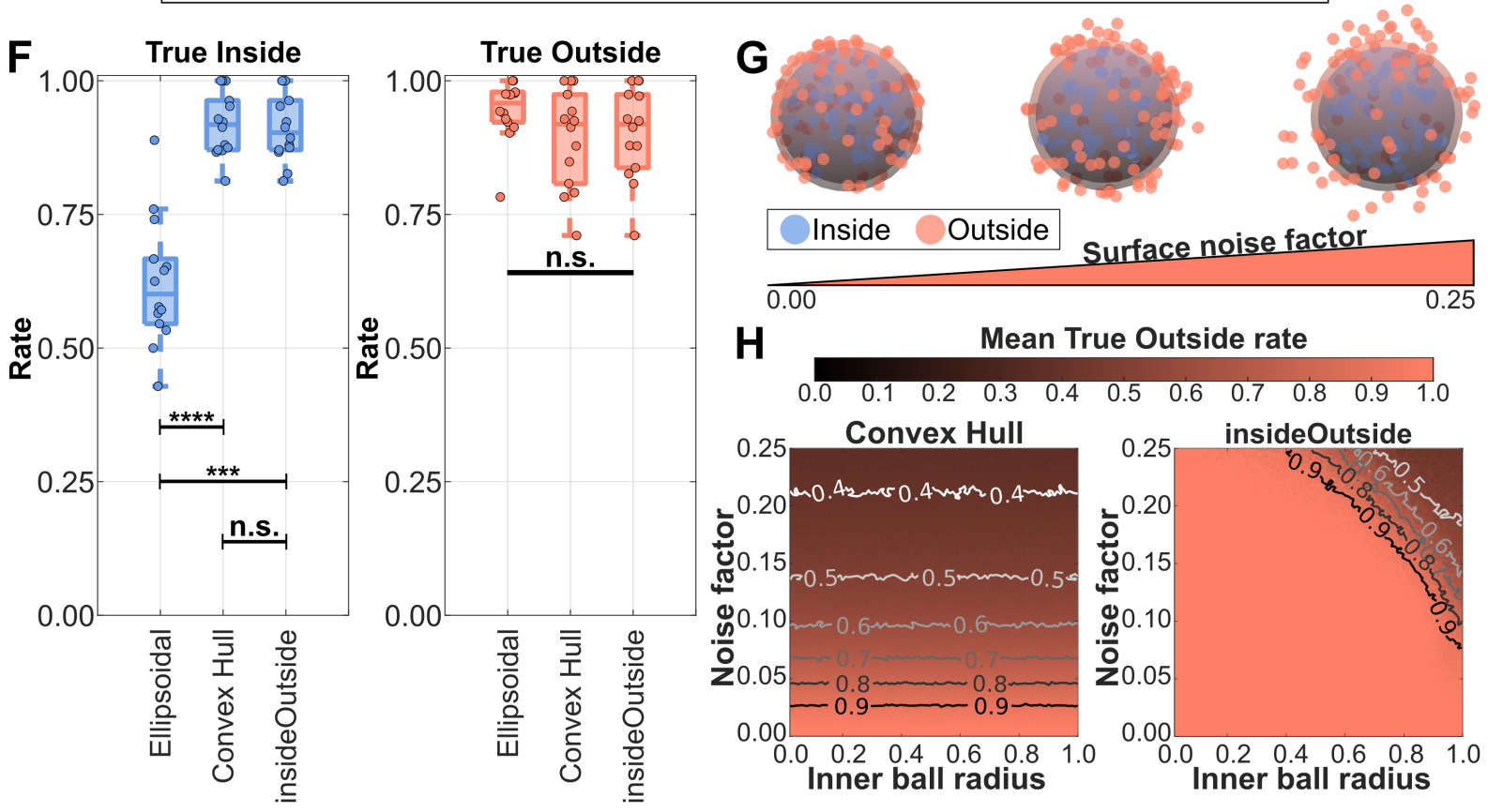

Figure 4: A. Confocal images of a mid-blastocyst stained for DNA and early ICM marker SOX2. A single slice is shown for transmitted light and maximum intensity projections are shown for fluorescence images. B. Gaussian mixture modelling (GMM) was performed on the SOX2 nuclear signal of 758 cells from 14 embryos to classify SOX2 positive (blue) and negative (orange) nuclei. Nuclear signal was normalized by nuclear volume, $\log _{10}$ transformation, and re-scaling to the interval [0,1]. C. GMM classification of cells applied to the embryo from A. D. SOX2 GMM classification of embryo from A shown in three-quarters view. SOX2 GMM classification was used as the ground truth for methods benchmarking. Shown are True Inside (blue) and True Outside (orange) cell classification. E. Classification of embryo from A by the Ellipsoidal (Lou et al., 2014), Convex Hull (Forsyth et al., 2021), and insideOutside (Stirparo 
et al. 2021) methods shown in three-quarter view. Shown are True Inside (blue), True Outside (orange), Misclassified Outside (green), and Misclassified Inside (gray) nuclei. F. Classification rates for True Inside (left, blue) and True Outside (right, orange). The Ellipsoidal method had a significantly lower True Inside classification rate than the Convex Hull method ( $\mathrm{p}$-value $=0.8 \times 10^{-4}$, Kruskal-Wallis) and the insideOutside algorithm ( $\mathrm{p}$-value $=1.3 \times 10^{-4}$, Kruskal-Wallis). All other pairwise relationships were not significant (n.s., p-values $\geq 0.63$, Kruskal-Wallis) G. Increasing levels of noise were added to the surface points of the test shape to simulate increasing local surface concavities. H. The mean True Outside rate (orange scale) is shown over the parameter space of inner ball radius (100 radii between 0.01 and 1) versus noise factor (100 levels between 0 and 0.25 ) for the Convex Hull (left) and insideOutside (right) methods. 100 test shapes were classified for each parameter pair. Additional contour lines are shown to delineate drops in classification rate.

\section{insideOutside has higher accuracy than the Convex Hull method when chal- lenged with local surface concavities}

While both the insideOutside and Convex Hull methods perform comparably on the empirical blastocysts, the Convex Hull method holds a systematic error of misclassifying outside points as inside points when small local surface concavities are introduced. We highlight this issue by emulating increasing levels of local surface concavities via the introduction of increasing levels of normally distributed random noise to the surface points of the test shapes (Figure $4 \mathrm{G}$ ). We then computed the classification rates over the parameter space of inner ball radius (100 radii between 0.01 and 1) and noise factor (100 levels between 0 and 0.25 ) for 100 shapes (Figure $4 \mathrm{H}$ and Figure S3A). The Convex Hull method shows a uniform decrease of True Outside classification rates across all inner ball radii for increasing levels of noise, eventually dropping below a rate of 0.4 around a noise factor greater than 0.2. Whereas the insideOutside method does not display this uniform decrease of True Outside classification rates where it maintains a rate of greater than 0.9 for the majority of the parameter sets tested. The insideOutside method only begins to lose accuracy when both the inner ball radius and noise factor become large. Finally we note that surface concavities have negligible effects on the classification rates of inside points for both methods (Figure $\mathrm{S} 3 \mathrm{~B}, \mathrm{C}$ ).

\section{Discussion}

Motivated by the need to accurately classify cells of mouse embryo by position alone, we present insideOutside, an accessible algorithm for the classification of interior and exterior points of a three-dimensional point-cloud. We established, both analytically and computationally, that for a convex shape, there exists an inverse relationship between the minimum distance to the shape's surface and variance in distances to the shape's surface. We then harnessed this inverse relationship to build an algorithm which allows for faithful classification of interior and exterior points by hierarchical clustering. We then proceeded to benchmark our method against two other published methods, Ellipsoidal (Lou et al., 2014) and Convex Hull (Forsyth et al., 2021), showing that the insideOutside method was as reliable or better at classifying nuclei of the pre-implantation mouse embryo. We closed by demonstrating that the insideOutside method 
has greater accuracy than the Convex Hull method in classifying exterior points when challenged with local surface concavities. Finally, we have packaged the algorithm as a stand-alone MATLAB function that takes in a set of points as an $n \times 3$ matrix that returns two outputs: an $n \times 1$ bit vector indexing inside, 0 , and outside, 1 , points and an $n \times 2$ matrix of the calculated parameter values of minimum distance and variance in distances to the surface generated from the input set of points This is freely available at https://github.com/stanleystrawbridge/insideOutside

We have shown here that the Convex Hull and insideOutside methods both outperform the Ellipsoidal method in the classification of interior nuclei of pre-implantation mouse embryos. However, all three methods perform comparably when classifying exterior nuclei. In fact, the Ellipsoidal method has the smallest variance in the classification of exterior nuclei. In all three methods we see that instances of misclassification are highest where the ICM is in contact with the TE (Figure $4 \mathrm{E}$ ). Moreover, we have shown, through simulation, that the insideOutside method has greater accuracy when challenged with surface concavities. This is of particular importance for classifying model systems whose exterior points exhibit high levels of noise. For example, columnar epithelium whose nuclei exhibit differential apiobasal polarity. These findings speak to the appropriateness of each method. There may be instances when the user has a large number of points in a low noise situation where exterior point should be strictly classified as belonging to the surface. In such a case the Convex Hull method is most appropriate. Alternatively, the user may want to soften this condition in the case of a small number of points in a high noise situation, e.g. the pre-implantation mouse embryo. This would indicate the use of the insideOutside method. While the Ellipsoidal method has proved useful in identifying unique embryos from images with many embryos (Lou et al., 2014), we would not recommend the Ellipsoidal method for classifying the nuclei of those embryos. Finally, we note that the insideOutside method is slightly slower than the Ellipsoidal and Convex Hull methods by virtue of it performing more calculations, i.e. a step in the insideOutside algorithm is the generation of a convex hull. However, this does not affect practical application and only becomes apparent when classifying numbers of shapes on the order of $>10^{6}$, as presented here in simulations.

There is scope for the refinement of the insideOutside algorithm. This could come by way of incorporating more information about the segmented nuclei, e.g. making use of nuclear aspect ratio and not just nuclear centroid. Additional parameters could also be introduced to the parameter decision space. IVEN has made use of number-of-neighbors, calculated from the Delaunay triangulation, in downstream spatial analysis. The addition of number-of-neighbors to the classification space may aid in better discrimination of interior and exterior points, especially in the problem case where the ICM meets the TE. Also the method of unsupervised clustering could be further explored. Here we present the insideOutside algorithm using hierarchical clustering with ward linkage, while k-means clustering was initially attempted. Many other unsupervised clustering methods exist (DBSCAN, spectral clustering, Gaussian mixture modelling, etc.) which could also be deployed.

We find it of particular importance to make this method, and future methods, easy-to-deploy for biological and life scientists, as there is increasing need for them to perform rigorous quan- 
tification of their data. Development of the insideOutside method in Stirparo et al. (2021) was born of a need to refine the original MINS classification method and was driven by collaboration between experimentalists and theoreticians. While there is no expectation for experimentalists to do methods development, there is expectation that they will be able to use these methods, thus empowering future work. Both MINS and IVEN share in this ethos of empowering experimentalists in the journey of data analysis. However, the insideOutside method stands apart from its counterpart methods in that it is a stand alone function, whereas the classification methods in MINS and IVEN are members of a larger software package. This means the insideOutside method has greater flexibility in use and migration to other programming languages. For example, implementations in open source languages like Python and $R$, which are widely used in the biological and life sciences, will make the insideOutside method more accessible as it would remove the dependency on a MATLAB licence. Critically, the stand-alone nature of the insideOutside method lends itself to incorporation into other software pipelines. Indeed, the insideOutside method could be incorporated as an additional classification method into either MINS or IVEN, as both packages have MATLAB implementations.

Finally, other use cases for the insideOutside method include other mammalian organisms that undergo the process of blastocyst formation (humans, non-human primates, other rodents, ungulates, etc.). It also has use for certain organoid systems, such as quantifying the level of cell sorting in ICM organoids (Mathew et al., 2019). And while the insideOutside method was motivated by the need to discriminate between the ICM and the TE in the pre-implantation blastocyst, it remains a general method for classifying the interior and exterior points of a point-cloud. This means it has extensibility to any data of this description. This includes, but is not limited to, the organization of transcription factor clusters from single-molecule localization microscopy (Liu et al., 2014), the pattern of RNA transcripts acquired through seq-FISH (Lohoff et al., 2021), and the relationship of genomic loci within the nucleus as determined by single-cell Hi-C structures (Stevens et al., 2017).

\section{Materials and Methods}

\section{Embryo collection and bright-field imaging}

Embryos were obtained from natural mating, detection of a copulation plug in the morning was used as confirmation of successful mating and indicated embryonic day (E) 0.5. 8-cell and compacted morula embryos were flushed from the oviduct at E2.5 and E3.0, respectively, and mid and late blastocysts were flushed from the uterine horns at E3.5 and E4.5, respectively, using M2 medium (Sigma-Aldrich, M7167). This research has been regulated under the Animals (Scientific Procedures) Act 1986 Amendment Regulations 2012 following ethical review by the University of Cambridge Animal Welfare and Ethical Review Body. Use of animals in this project was approved by the ethical review committee for the University of Cambridge, and relevant Home Office licences (Project licence No. 80/2597 and No. P76777883) are in place. Bright-field images were taken on a Leica DMI4000B microscope. 


\section{Quantitative immunofluorescence of embryos}

Quantitative immunofluorescence data was originally published in Stirparo et al. (2021). In brief, embryos were fixed in paraformaldehyde, stained for DNA and SOX2, and imaged using confocal microscopy. Embryo nuclei were segmented and quantified using MINS (Lou et al. 2014) to calculate the nuclear parameters of total fluorescence (sum of pixel values), volume, and centroid.

\section{Code availability}

The insideOut algorithm and all code used in this manuscript to perform simulations, analysis, and benchmarking are written in MATLAB (2021a) and are freely available at https://github.com/stanleystrawbridge/insideOutside under the GNU General Public License v3.0.

\section{Acknowledgements}

The authors thank members of the Nichols and Fletcher groups for helpful feedback in the preparation of the manuscript. Collaboration between the Nichols and Fletcher groups was made possible through a Company of Biologists Travelling Fellowship awarded to SES (DEVTF180513). AGF acknowledges support from the Biotechnology and Biological Sciences Research Council (BB/V018647/1 and BB/R016925/1).

\section{Author contributions}

SES conceived and developed the insideOutside algorithm. ECS collected bright field images of pre-implantation mouse embryos. SES and AGF performed mathematical analyses. AK performed embryo immunostainings and segmentation used for methods benchmarking. SES performed simulations and computational analyses. SES, AGF, and JN wrote the manuscript.

\section{References}

Arnold, K., Sarkar, A., Yram, M. A., Polo, J. M., Bronson, R., Sengupta, S., Seandel, M., Geijsen, N., and Hochedlinger, K. (2011). Sox2+ adult stem and progenitor cells are important for tissue regeneration and survival of mice. Cell stem cell, 9(4):317-329.

Barber, C. B., Dobkin, D. P., and Huhdanpaa, H. (1996). The quickhull algorithm for convex hulls. ACM Transactions on Mathematical Software (TOMS), 22(4):469-483.

Bessonnard, S., De Mot, L., Gonze, D., Barriol, M., Dennis, C., Goldbeter, A., Dupont, G., and Chazaud, C. (2014). Gata6, nanog and erk signaling control cell fate in the inner cell mass through a tristable regulatory network. Development, 141(19):3637-3648. 
Blin, G., Sadurska, D., Portero Migueles, R., Chen, N., Watson, J., and Lowell, S. (2019). Nessys: a new set of tools for the automated detection of nuclei within intact tissues and dense 3D cultures. PLoS Biol., 17(8):e3000388.

Boroviak, T., Stirparo, G. G., Dietmann, S., Hernando-Herraez, I., Mohammed, H., Reik, W., Smith, A., Sasaki, E., Nichols, J., and Bertone, P. (2018). Single cell transcriptome analysis of human, marmoset and mouse embryos reveals common and divergent features of preimplantation development. Development, 145(21):dev167833.

Chazaud, C., Yamanaka, Y., Pawson, T., and Rossant, J. (2006). Early lineage segregation between epiblast and primitive endoderm in mouse blastocysts through the grb2-mapk pathway. Developmental cell, 10(5):615-624.

Deserno, M. (2004). How to generate equidistributed points on the surface of a sphere. https: //www.cmu.edu/biolphys/deserno/pdf/sphere_equi.pdf. [Online; accessed: 10.02.2021].

Evans, M. J. and Kaufman, M. H. (1981). Establishment in culture of pluripotential cells from mouse embryos. nature, 292(5819):154-156.

Fleming, T. P. (1987). A quantitative analysis of cell allocation to trophectoderm and inner cell mass in the mouse blastocyst. Dev. Biol., 119(2):520-531.

Forsyth, J., Al-Anbaki, A., de la Fuente, R., Modare, N., Perez-Cortes, D., Rivera, I., Kelly, R., Cotter, S., and Plusa, B. (2021). IVEN: A quantitative tool to describe 3D cell position and neighbourhood reveals architectural changes in FGF4-treated preimplantation embryos. PLoS Biol., 19(7):e3001345.

Gardner, R. and Johnson, M. (1972). An investigation of inner cell mass and trophoblast tissues following their isolation from the mouse blastocyst. J.Embryol.exp.Morph.

Gardner, R. and Rossant, J. (1979). Investigation of the fate of 4.5 day post-coitum mouse inner cell mass cells by blastocyst injection. J.Embryol.exp.Morph.

Grabarek, J. B., Żyżyńska, K., Saiz, N., Piliszek, A., Frankenberg, S., Nichols, J., Hadjantonakis, A.-K., and Plusa, B. (2012). Differential plasticity of epiblast and primitive endoderm precursors within the icm of the early mouse embryo. Development, 139(1):129-139.

Guo, F., Li, L., Li, J., Wu, X., Hu, B., Zhu, P., Wen, L., and Tang, F. (2017). Single-cell multi-omics sequencing of mouse early embryos and embryonic stem cells. Cell research, 27(8):967-988.

Guo, G., Huss, M., Tong, G. Q., Wang, C., Sun, L. L., Clarke, N. D., and Robson, P. (2010). Resolution of cell fate decisions revealed by single-cell gene expression analysis from zygote to blastocyst. Developmental cell, 18(4):675-685. 
Guo, G., Stirparo, G. G., Strawbridge, S. E., Spindlow, D., Yang, J., Clarke, J., Dattani, A., Yanagida, A., Li, M. A., Myers, S., et al. (2021). Human naive epiblast cells possess unrestricted lineage potential. Cell stem cell, 28(6):1040-1056.

Hamilton, T. G., Klinghoffer, R. A., Corrin, P. D., and Soriano, P. (2003). Evolutionary divergence of platelet-derived growth factor alpha receptor signaling mechanisms. Molecular and cellular biology, 23(11):4013-4025.

Johnson, M. H. and Ziomek, C. A. (1981). The foundation of two distinct cell lineages within the mouse morula. Cell, 24(1):71-80.

Kalkan, T., Olova, N., Roode, M., Mulas, C., Lee, H. J., Nett, I., Marks, H., Walker, R., Stunnenberg, H. G., Lilley, K. S., et al. (2017). Tracking the embryonic stem cell transition from ground state pluripotency. Development, 144(7):1221-1234.

Kurimoto, K., Yabuta, Y., Ohinata, Y., Ono, Y., Uno, K. D., Yamada, R. G., Ueda, H. R., and Saitou, M. (2006). An improved single-cell cdna amplification method for efficient high-density oligonucleotide microarray analysis. Nucleic acids research, 34(5):e42-e42.

Lawson, K. A., Dunn, N. R., Roelen, B. A., Zeinstra, L. M., Davis, A. M., Wright, C. V., Korving, J. P., and Hogan, B. L. (1999). Bmp4 is required for the generation of primordial germ cells in the mouse embryo. Genes $\& 5$ development, 13(4):424-436.

Le Bin, G. C., Muñoz-Descalzo, S., Kurowski, A., Leitch, H., Lou, X., Mansfield, W., EtienneDumeau, C., Grabole, N., Mulas, C., Niwa, H., et al. (2014). Oct4 is required for lineage priming in the developing inner cell mass of the mouse blastocyst. Development, 141(5):10011010.

Liu, Z., Legant, W. R., Chen, B.-C., Li, L., Grimm, J. B., Lavis, L. D., Betzig, E., and Tjian, R. (2014). 3d imaging of sox2 enhancer clusters in embryonic stem cells. elife, 3:e04236.

Lohoff, T., Ghazanfar, S., Missarova, A., Koulena, N., Pierson, N., Griffiths, J., Bardot, E., Eng, C.-H., Tyser, R., Argelaguet, R., et al. (2021). Integration of spatial and single-cell transcriptomic data elucidates mouse organogenesis. Nature biotechnology, pages 1-12.

Lou, X., Kang, M., Xenopoulos, P., Munoz-Descalzo, S., and Hadjantonakis, A.-K. (2014). A rapid and efficient 2D/3D nuclear segmentation method for analysis of early mouse embryo and stem cell image data. Stem Cell Rep., 2(3):382-397.

Malter, H. E. and Cohen, J. (1989). Blastocyst formation and hatching in vitro following zona drilling of mouse and human embryos. Gamete research, 24(1):67-80.

Martin, G. R. (1981). Isolation of a pluripotent cell line from early mouse embryos cultured in medium conditioned by teratocarcinoma stem cells. Proceedings of the National Academy of Sciences, 78(12):7634-7638. 
Mathew, B., Muñoz-Descalzo, S., Corujo-Simon, E., Schröter, C., Stelzer, E. H., and Fischer, S. C. (2019). Mouse icm organoids reveal three-dimensional cell fate clustering. Biophysical journal, 116(1):127-141.

McDole, K. and Zheng, Y. (2012). Generation and live imaging of an endogenous cdx2 reporter mouse line. Genesis, 50(10):775-782.

Morgani, S. M., Saiz, N., Garg, V., Raina, D., Simon, C. S., Kang, M., Arias, A. M., Nichols, J., Schröter, C., and Hadjantonakis, A.-K. (2018). A sprouty4 reporter to monitor fgf/erk signaling activity in escs and mice. Dev. Biol., 441(1):104-126.

Nichols, J. and Gardner, R. (1984). Heterogeneous differentiation of external cells in individual isolated early mouse inner cell masses in culture. J.Embryol.exp.Morph.

Niwa, H., Toyooka, Y., Shimosato, D., Strumpf, D., Takahashi, K., Yagi, R., and Rossant, J. (2005). Interaction between oct $3 / 4$ and cdx2 determines trophectoderm differentiation. Cell, 123(5):917-929.

Palmieri, S. L., Peter, W., Hess, H., and SCHöLER, H. R. (1994). Oct-4 transcription factor is differentially expressed in the mouse embryo during establishment of the first two extraembryonic cell lineages involved in implantation. Developmental biology, 166(1):259-267.

Plusa, B., Piliszek, A. E., Frankenberg, S., Artus, J., and Hadjantonakis, A.-K. (2008). Distinct sequential cell behaviours direct primitive endoderm formation in the mouse blastocyst. Dev. Biol., 2(319):537-538.

Saiz, N., Mora-Bitria, L., Rahman, S., George, H., Herder, J. P., García-Ojalvo, J., and Hadjantonakis, A.-K. (2020). Growth-factor-mediated coupling between lineage size and cell fate choice underlies robustness of mammalian development. Elife, 9:e56079.

Saiz, N., Williams, K. M., Seshan, V. E., and Hadjantonakis, A.-K. (2016). Asynchronous fate decisions by single cells collectively ensure consistent lineage composition in the mouse blastocyst. Nat. Commun., 7(1):1-14.

Schindelin, J., Arganda-Carreras, I., Frise, E., Kaynig, V., Longair, M., Pietzsch, T., Preibisch, S., Rueden, C., Saalfeld, S., Schmid, B., et al. (2012). Fiji: an open-source platform for biological-image analysis. Nat. Meth., 9(7):676-682.

Schrode, N., Saiz, N., Di Talia, S., and Hadjantonakis, A.-K. (2014). Gata6 levels modulate primitive endoderm cell fate choice and timing in the mouse blastocyst. Developmental cell, 29(4):454-467.

Smith, R. and McLaren, A. (1977). Factors affecting the time of formation of the mouse blastocoele. J.Embryol.exp.Morph. 
Stevens, T. J., Lando, D., Basu, S., Atkinson, L. P., Cao, Y., Lee, S. F., Leeb, M., Wohlfahrt, K. J., Boucher, W., O'Shaughnessy-Kirwan, A., et al. (2017). 3d structures of individual mammalian genomes studied by single-cell hi-c. Nature, 544(7648):59-64.

Stirparo, G., Kurowski, A., Yanagida, A., Bates, L., Strawbridge, S., Hladkou, S., Stuart, H., Boroviak, T., Silva, J., and Nichols, J. (2021). OCT4 induces embryonic pluripotency via STAT3 signaling and metabolic mechanisms. Proc. Natl. Acad. Sci. U.S.A., 118(3):e2008890118.

Stirparo, G. G., Boroviak, T., Guo, G., Nichols, J., Smith, A., and Bertone, P. (2018). Integrated analysis of single-cell embryo data yields a unified transcriptome signature for the human preimplantation epiblast. Development, 145(3):dev158501.

Suzuki, H., Togashi, M., Adachi, J., and Toyoda, Y. (1995). Developmental ability of zona-free mouse embryos is influenced by cell association at the 4-cell stage. Biology of reproduction, 53(1):78-83.

Tarkowski, A. K. and Wróblewska, J. (1967). Development of blastomeres of mouse eggs isolated at the 4-and 8-cell stage. J.Embryol.exp.Morph.

Wicklow, E., Blij, S., Frum, T., Hirate, Y., Lang, R., Sasaki, H., and Ralston, A. (2014). HIPPO pathway members restrict SOX2 to the inner cell mass where it promotes ICM fates in the mouse blastocyst. PLoS Genet., 10(10):e1004618.

Yoshinaga, K., Meyer, R. K., and Greep, R. O. (1976). Implantation of the Ovum. Harvard University Press.

Ziomek, C. and Johnson, M. (1980). Cell surface interaction induces polarization of mouse 8-cell blastomeres at compaction. Cell, 21(3):935-942. 


\section{Supplemental material}

\section{Uniform random points on a sphere}
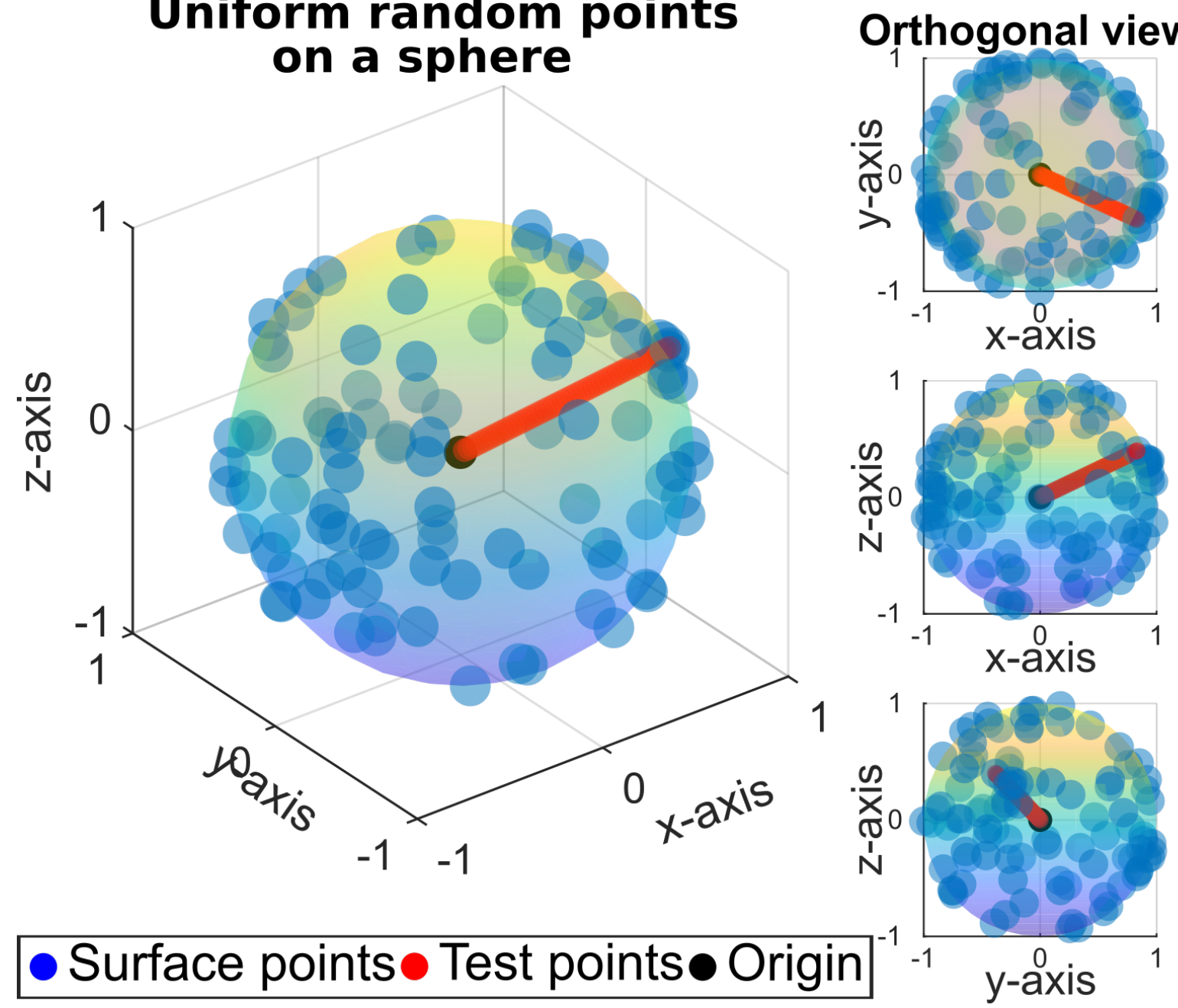

Figure S1: Example of 100 uniform random points (blue dots) plotted on the unit sphere (rainbow surface). The minimum distance to the surface, $m$, and variance in distances to the surface, $v$, are calculated for 50 test points (red dots) along the vector from the origin (black dot) to a random point on the surface. Shown are the three-quarters view (left) and the three orthogonal views (right). 1000 simulations were performed for the data in Figure $2 \mathrm{E}$. 

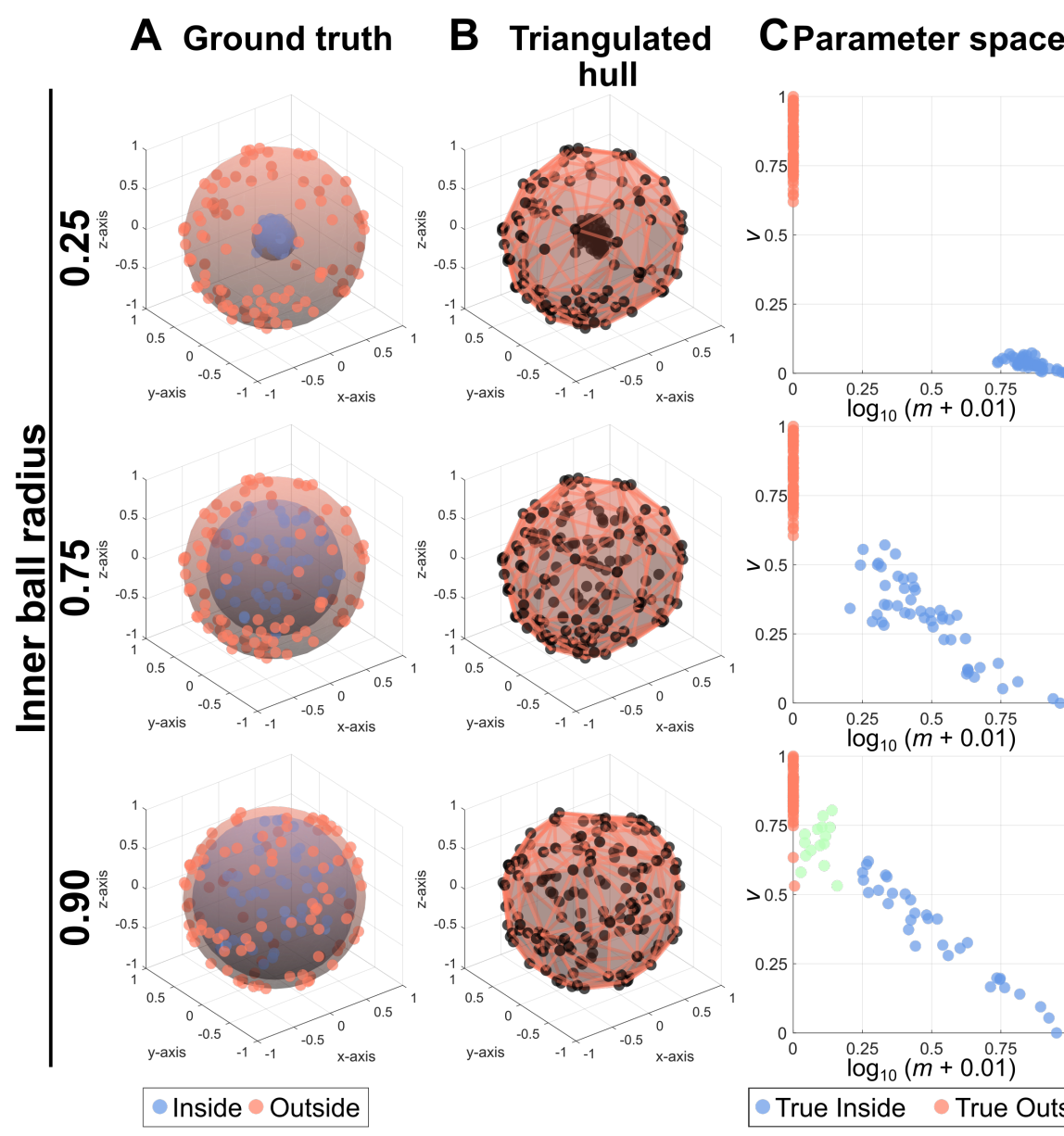

\section{Classification}
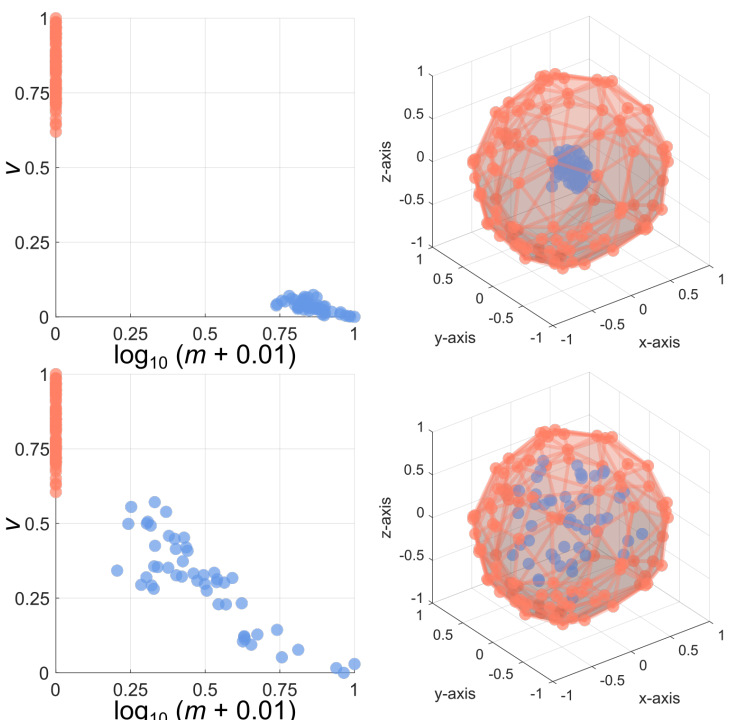

Figure S2: Example shapes with different inner ball radii used for accuracy testing. A. Ground truth of inside points, blue dots enclosed by blue surface, and outside points, orange points on orange surface. B. The triangulated hull generated from making a convex hull over the Delaunay triangulation. C. The classification of points using hierarchical clustering over the calculated parameter space. Shown are True Inside points (blue), True Outside points (orange), and Misclassified Outside points (green). D. The classification mapped onto the original shape. 

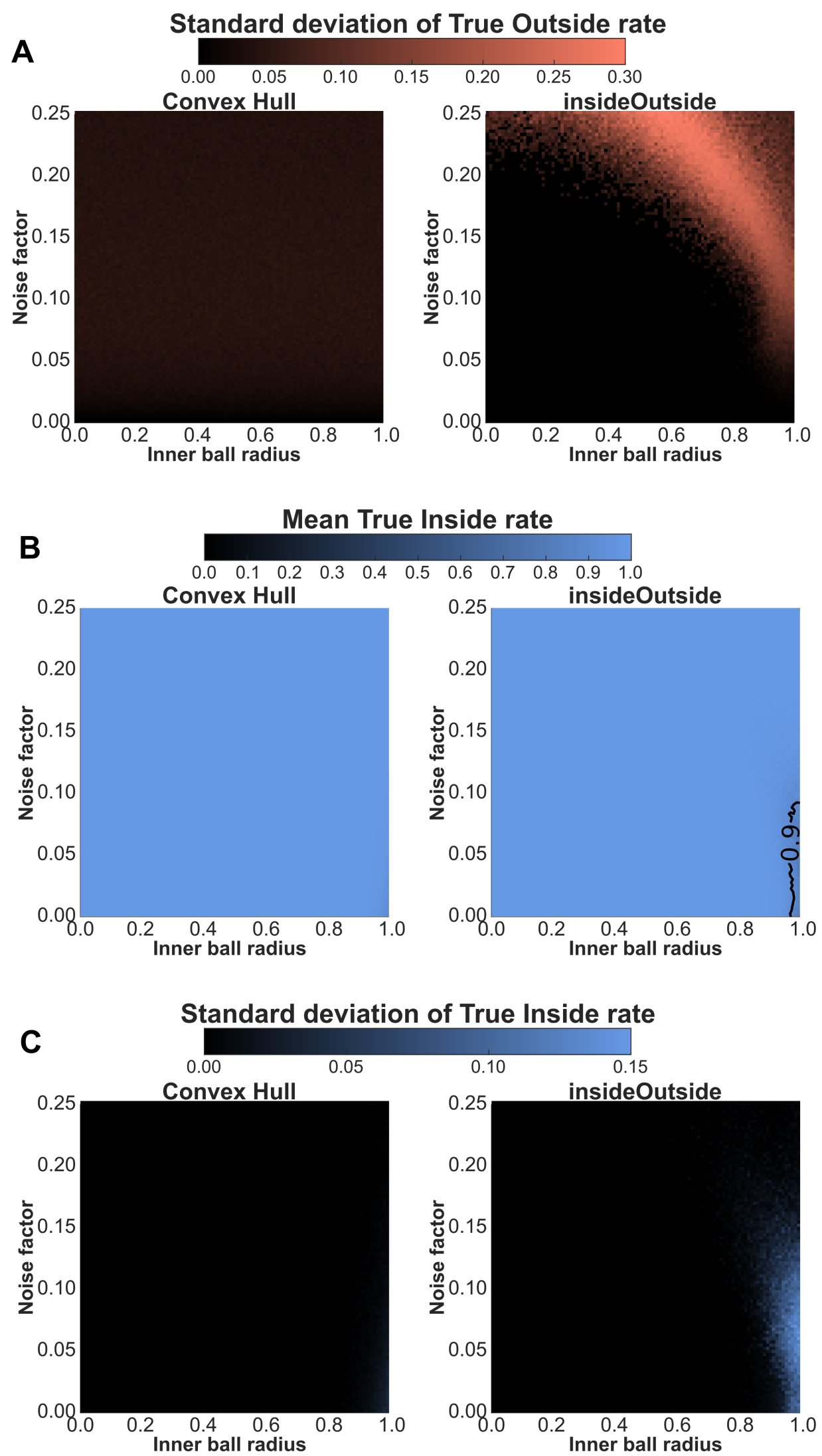
tween 0.01 and 1) versus noise factor (100 levels between 0 and 0.25) for the Convex Hull (left) and insideOutside (right) methods. A. The standard deviation of the True Outside rate. B. The mean True Outside rate. Additional contour lines are shown to delineate drops in classification rate (everywhere $>0.9$ except near $[1,0])$. C. The standard deviation of the True Inside rate. 